\title{
DESARROLLO DE UN SISTEMA DE INFORMACIÓN GEOGRÁFICA PARA MEJORAR LA GESTIÓN DEL AGUA DE RIEGO DEL EMBALSE CONVENTO VIEJO, CHILE.
}

\author{
Development of a geographic information system to improve the irrigation \\ water management of the Convento Viejo Dam, Chile.
}

\author{
Carlos Mena $^{1 *}$, Yony Ormazábal ${ }^{1}$, José Luis Llanos ${ }^{2}$, José Díaz $^{3}$
}

\begin{abstract}
A B S T R A C T
When establishing strategic lines for agricultural activities, geomatic and its diverse technologies allow generating a highly representative and reliable characterization of the territory, expressed in a dynamic and flexible digital data base. This helps the planning and monitoring of the productive changes observed in lands benefited with the increase of irrigation water and the improvement of its distribution network. The main goal of the investigation was to create a Geographic Information System (GIS), feed it with farming and socioeconomic information bases allowing the identification of homogeneous intervention sectors and the establishment of an agricultural exploitation typology of the zones directly or indirectly benefited by the construction of the "Convento Viejo" Dam in its second stage. The methodology employed included the creation of a digital database from the compilation and processing of different sources and formats of digital geographic cover, geo-processing of the covers to identify homogenous intervention zones, design and application of a survey to classify the agricultural exploitations and the elaboration of a GIS consultation interphase. Four homogeneous geographic sectors were identified and delimited by topographic, agricultural and climatic variables. The agricultural exploitations typology resulted in four conglomerates (clusters) grouped according to social, productive, financial and commercial variables. The designed GIS allows easy consultation and analysis of the information improving agricultural management and planning.
\end{abstract}

Key words: dam, geomatic, irrigation water management, digital data base, geographic information system.

\section{R E S U M E N}

Dentro del proceso de establecimiento de las líneas de desarrollo estratégico para la actividad agrícola, la geomática y sus diversas tecnologías permiten generar una caracterización altamente representativa y confiable del territorio, expresada en una base de datos digital dinámica y flexible, que propicia la planificación y el seguimiento de los cambios productivos observados en los terrenos beneficiados con el incremento del agua de riego y el mejoramiento de su red de distribución. El objetivo principal de la investigación fue crear un Sistema de Información Geográfica (SIG), alimentado con información base agropecuaria y socioeconómica, que permita la identificación de sectores homogéneos de intervención y el establecimiento de la tipología de las explotaciones agrícolas de las zonas directa o indirectamente beneficiadas con la construcción del Embalse Convento Viejo en su segunda etapa. La metodología empleada consideró la creación de una base de datos digital a partir de la recopilación y procesamiento de coberturas geográficas digitales de distintas fuentes y formatos, el geoprocesamiento de coberturas para identificar zonas homogéneas de intervención, el diseño y aplicación de una encuesta para tipificar las explotaciones agrícolas, y la elaboración de una interfase de consulta SIG. Se identificaron cuatro sectores geográficos homogéneos delimitados por variables topográficas y agroclimáticas. La tipología de las explotaciones agrícolas arrojó cuatro conglomerados (clusters) agrupados de acuerdo a variables sociales, productivas, financieras y comerciales. El SIG diseñado permite consultar y analizar fácilmente la información, mejorando la gestión y planificación agrícola.

Palabras clave: embalse, geomática, gestión del agua de riego, base de datos digital, sistema de información geográfica.

\footnotetext{
${ }^{1}$ Universidad de Talca, Facultad de Ciencias Forestales, Casilla 721, Talca, Chile.

E-mail:cmena@utalca.cl*Autor para correspondencia.

${ }^{2}$ Universidad de Santiago de Chile, Facultad Tecnológica, Av. Ecuador 3769, Santiago, Chile. E-mail: jllanos@usach.cl

${ }^{3}$ Universidad de Talca, Facultad de Ciencias Agrarias, Casilla 721, Talca, Chile. E-mail: jdiazoso@utalca.cl

Recibido: 9 de diciembre de 2005. Aceptado: 6 de abril de 2006.
} 


\section{INTRODUCCIÓN}

La consolidación de la agricultura de riego continúa siendo un aspecto prioritario, tanto para países desarrollados como en vías de desarrollo. Generalmente, el crecimiento sostenible de la agricultura de riego se ve limitado por escasez de agua y de tierras regables (Manz, 1994). Sin embargo, existe la posibilidad de incrementar en forma importante la producción agrícola si se utilizan eficientemente el agua y la infraestructura disponibles, mediante un mejor manejo de los sistemas de conducción (incluyendo la operación), modernización de la infraestructura, un mejor mantenimiento y prácticas agrícolas más adecuadas (Sagardoy, 2003). De hecho, el nuevo escenario conformado por dichas mejoras, modificará significativamente la estructura productiva de la zona beneficiada, lo cual conlleva a un incremento en la cantidad y la calidad de la producción local, la generación de nuevas fuentes de trabajo y el aumento sustancial de los ingresos del sector.

Si bien el desarrollo y viabilidad de proyectos de riego desde los puntos de vista económico y social dependerá fuertemente de los factores mencionados, en el último tiempo la disponibilidad de tecnología e informática ofrecen nuevas herramientas que acompañan, potencian y enriquecen los procesos llevados a cabo. Específicamente la geomática, a través de los Sistemas de Información Geográfica (SIG), la teledetección y el Sistema de Posicionamiento Global (GPS), están tomando un rol preponderante en el manejo de los recursos hídricos, al conjugar la dimensión espacial y temática en la representación precisa de los componentes territoriales (Bastiaanssen et al., 2003), elementos claves al momento de configurar un escenario ajustado a la realidad en donde cada componente presenta características propias que pueden y deben ser analizadas conjuntamente. La potencialidad de riego ha sido usada en muchas ocasiones como el único criterio para establecer las políticas agrícolas y de recursos hídricos de un país, sin hacer un análisis integrado de las limitaciones económicas, sociales, institucionales, ambientales y de mercados. En este contexto, los malos resultados de algunos proyectos de riego en la producción agrícola pueden ser atribuidos sin duda a una percepción limitada o errónea de la infraestructura de riego y de la distribución de agua (en sus dimensiones espaciales y temáticas), y no a la productividad de los sistemas de producción y su capacidad de respuesta a los mercados agropecuarios (Burke, 2003).
La generación de información temática y espacial mediante herramientas geomáticas, permite la conformación de un completo y preciso escenario territorial que favorece el análisis y comprensión de las múltiples variables que determinan el potencial productivo base para establecer esquemas de producción agrícola adecuados a las condiciones existentes. En este contexto, la determinación de sectores homogéneos de intervención mediante geoprocesamiento, permite el análisis conjunto e integrado de variables territoriales factibles de ser expresadas espacialmente, lo cual constituye el punto de partida necesario para establecer una tipología de explotaciones que oriente las líneas de acción en un nuevo escenario con mayor y mejor disponibilidad del recurso agua (Bastiaanssen et al., 2003).

Entre las diversas investigaciones que se han realizado al respecto, se puede mencionar aquella llevada a cabo en la Provincia de Almería, España, destinada a mejorar la gestión de la distribución de agua mediante redes colectivas de tuberías para riego en zonas con cultivos hortícolas intensivos (Zapata et al., 2000). En dicho estudio, se elaboró una completa base cartográfica digital que reflejaba la situación real de la red de riego, con lo cual fue posible realizar un análisis detallado de la misma, como también de la gestión de la zona regable. Con la utilización de herramientas tecnológicas de diseño, simulación y manejo de los sistemas de distribución, se generó una reducción en la inversión y en los costos de explotación, así como un aumento de la producción agrícola, por lo que los primeros beneficiados fueron los gestores y usuarios de dichos sistemas de riego, principalmente agricultores. En esta misma línea, un estudio realizado en la Cuenca del río Indo en Pakistán, utilizó sensores remotos y tecnología SIG para evaluar la productividad del agua de varios cultivos con distintos sistemas de riego, y demostró que la productividad del agua en un cultivo varía considerablemente en función de la escala de estudio (canales pequeños y canales mayores) y de la distribución de las áreas de riego consideradas (Bastiaanssen et al., 2003).

El proyecto de ampliación del Embalse Convento

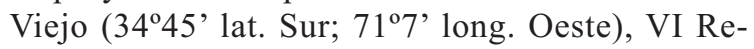
gión de Chile, significará aumentar su capacidad de regulación de aguas de 27 millones a 237 millones de $\mathrm{m}^{3}$, además de extender en $300 \mathrm{~km}$ la actual 
red de canales de riego, todo lo cual involucrará una inversión en obras de aproximadamente 105 millones de dólares. Este nuevo escenario presenta grandes oportunidades para los propietarios agrícolas de las zonas beneficiadas con la ampliación, las cuales sólo se concretarán diseñando e implementando planes de gestión dirigidos a potenciar sistemas de producción adecuados para cada zona, en donde el conocimiento preciso de las condiciones ambientales, sociales y productivas de las zonas a intervenir es fundamental para la correcta toma de decisiones. En este contexto y considerando la amplitud de la zona de estudio, se planteó como objetivo principal crear un Sistema de Información Geográfica (SIG), alimentado con información base agropecuaria y socioeconómica, que permita la identificación de sectores homogéneos de intervención y el establecimiento de la tipología de las explotaciones agrícolas de las zonas directa o indirectamente beneficiadas con la construcción del Embalse Convento Viejo en su segunda etapa.

\section{MATERIALES Y MÉTODOS}

\section{Localización geográfica y descripción de la zona de estudio}

El estudio se localiza en la zona comprendida por las comunas de Lolol, Pumanque, Chépica, Santa Cruz, Palmilla y Peralillo, cubriendo un área de 96.506 ha $\left(34^{\circ} 11^{\prime}\right.$ ' a $34^{\circ} 58^{\prime}$ lat. Sur; $70^{\circ} 47^{\prime}$ a $71^{\circ} 54^{\prime}$ long. Oeste). La zona presenta notables ventajas del régimen de radiación solar y temperatura, por lo cual es posible el desarrollo de rotaciones de cultivos de alta intensidad de uso del suelo. La acumulación de horas-frío y los altos niveles de acumulación calóricas (días-grado) en primavera y verano, permiten el desarrollo de una amplia gama de especies frutícolas, incluidas las uvas de exportación y la producción de vinos de alto potencial de exportación. En general, en el área prácticamente no existen suelos con limitaciones para cultivos anuales y semipermanentes.

En cuanto a la estructura de la propiedad de la tierra, se puede indicar que 4.884 explotaciones corresponden a pequeños propietarios (29.705 ha), 1.463 explotaciones corresponden a medianos propietarios (25.053 ha) y 264 explotaciones son grandes propietarios (41.729 ha) (INE, 1997). Del total de la población, un $61,6 \%$ tiene menos de 44 años de edad y un $54,6 \%$ corresponde a población rural (INE, 2000).

\section{Confección de la base de datos digital para el área de estudio}

Para la confección de la base de datos digital para el área de estudio, se contempló la readecuación de la Base de Datos de Riego existente en la Comisión Nacional de Riego (CNR), tanto de sus componentes geográficos como temáticos, la incorporación de información geográfica existente en otros formatos y en distintas fuentes, además de la generación de información derivada y la recolección de información en terreno. Todo lo anterior implicó la realización de las siguientes fases:

- Transformación y estandarización de formatos digitales: comprendió la exportación digital de información geográfica desde el formato de origen al formato nativo del software ArcView (shape) (Hutchinson y Daniel, 2000), estandarizando y asignando un nombre de archivo para cada cobertura (shape).

- Adecuación y compatibilización de escala: implicó el análisis de las distintas coberturas de la base de datos, para establecer la compatibilidad de escalas. En los casos donde existieron diferencias de escala importantes, se optó buscar una fuente alternativa de información o desechar el uso de esa información. Se asumió 1:50.000 como escala de trabajo estándar para toda la base de datos.

- Incorporación de información geográfica procedente de distintas fuentes disponibles: se incorporó a la base de datos información disponible en formatos distintos. Las coberturas más importantes fueron:

a. Imagen Landsat año 2002, corregida en base a vectores (hidrografía y caminos) y recortada a la zona de estudio. El procesamiento se hizo en el software PCI Geomatics (Zhang et al., 2005) y el resultado fue un archivo de imagen IMG (image format) de resolución $30 \mathrm{~m}$, compatible con el software ArcView, lo que posibilita visualización como imagen de fondo para el resto de las coberturas vectoriales de la base.

b. Fotografías aéreas verticales CONAMA año 2001, escala 1:115.000, corregidas individualmente en base a vectores (hidrografía y caminos). La resolución final de cada fotografía fue de $15 \mathrm{~m}$. Finalmente, se procedió a realizar un mosaico con las 30 fotografías corregidas, obteniendo un archivo final de imagen de fondo en formato IMG, el cual se incluyó dentro de la base. 
- Actualización de la información geográfica: en base a la imagen del mosaico de fotografías se vectorizaron los caminos que no aparecían en la cobertura digital IGM (Instituto Geográfico Militar), por medio de las rutinas de edición del software ArcView. De igual forma, se actualizaron los polígonos correspondientes a las zonas urbanas presentes en la zona de estudio. El proceso se complementó con levantamientos GPS en terreno, en los cuales además se recopilaron los topónimos correspondientes a poblados.

- Edición digital de información: mediante herramientas de edición del software ArcInfo (Mena, 2005), se corrigieron los errores gráficos y temáticos presentes en cada una de las coberturas incorporadas a la base de datos, tales como líneas discontinuas, polígonos mal cerrados, nodos colgantes y registros inexistentes. Además, en el mismo software se construyeron las relaciones topológicas de cada elemento (conectividad, adyacencia y coincidencia), lo cual permite una mejor modelación digital de los datos.

- Verificación de información en terreno: se llevó a cabo una verificación de la información por medio de inspecciones visuales en terreno con apoyo de equipo GPS (Trimble, model Pro-XR, Dyton, Ohio, EE.UU.). Para esto se elaboró cartografía de detalle sobre la cual se planificaron los recorridos efectuados y a la vez, sirvió de soporte para la anotación de observaciones o anomalías detectadas en terreno que debieron ser corregidas posteriormente en la base.

- Análisis y generación de información topográfica derivada: se generó un modelo digital de elevaciones (DEM) a partir de la cobertura de curvas de nivel (equidistancia $25 \mathrm{~m}$ ), por medio del módulo "surface interpolation" del software Idrisi32 (Van Den Eeckhaut et al., 2005). Se trabajó con un tamaño de píxel de $50 \mathrm{~m}$, utilizando el algoritmo Consurf modificado (Douglas, 2000). Desde el DEM obtenido, se extrajeron las coberturas de pendientes, altimetría y orientación, utilizando el módulo Topographic variables de Idrisi32. Finalmente, se realizó un análisis de influencia en torno a los elementos geográficos de cursos de agua, caminos y poblados, en donde la distancia entre los anillos concéntricos fue de $500 \mathrm{~m}$. Todas las coberturas obtenidas en esta etapa, se transformaron a formato "shape", mediante el módulo Export de Idrisi32.
- Validación de información geográfica: se validó el DEM generado, por medio de la toma de puntos de control GPS en terreno. Se efectuaron sesiones de medición dinámica, configurando el georreceptor para toma rápida en código y fase portadora. De estas sesiones se obtuvieron 859 puntos medidos con precisión de $40 \mathrm{~cm}$, los cuales se utilizaron para contrastar el valor altimétrico registrado en terreno con el valor altimétrico indicado en el DEM para la misma posición. El error medio cuadrático (RSM) obtenido fue igual a $10,8 \mathrm{~m}$, lo cual es apropiado para la escala de trabajo de la base (FGDC, 1998).

\section{Determinación de sectores geográficos homogé- neos de intervención}

La identificación de sectores geográficos homogéneos de intervención se realizó a partir de la base de datos digital confeccionada para la zona de estudio, considerando principalmente, las capas de información relacionadas con los distritos agroclimáticos presentes en la zona, la capacidad de uso potencial y el drenaje del suelo, pendientes y orientaciones, la red hidrográfica superficial y la red de canales de riego proyectada. También se incluyeron aspectos como límites político-administrativos y la delimitación de cuencas y subcuencas.

Una vez establecidas las coberturas a incluir en el proceso, se procedió a verificar las características técnicas de las mismas, de manera de asegurar la compatibilidad de archivos, formatos y escalas. En la delimitación de los sectores geográficos homogéneos se emplearon funciones de geoprocesamiento propias de los SIG, tales como: intersección de elementos, intersección de coberturas, recorte de coberturas basado en otra cobertura o elementos de ésta, fusión de coberturas, superposición o conjunción de coberturas, etc. El proceso involucró procesos entre los elementos de un mismo archivo, así como entre coberturas digitales distintas. Como resultado, se obtuvieron nuevas coberturas digitales que representan a los sectores homogéneos en cuanto a sus características internas y su relación con los demás temas. Para lograr una correcta utilización de la información y obtener el máximo de beneficio, se adecuó la base de datos temática asociada a cada una de las coberturas obtenidas, de manera de caracterizar cada área homogénea. 


\section{Establecimiento de la tipología de las explota- ciones agrícolas}

Para el establecimiento de la tipología de las explotaciones agrícolas presentes en el área de estudio, se tomó como base al conjunto de explotaciones censadas los años 1996-1997, en el último censo agropecuario realizado por el Instituto Nacional de Estadísticas (INE, 1997). Dicha base censal quedó claramente representada como una capa más de información (capa de roles censales) de la base de datos digital de la zona en estudio, que sirvió de punto de partida para la aplicación de una encuesta a un número representativo de explotaciones agrícolas. El muestreo utilizado en la aplicación de la encuesta se segmentó en función de dos criterios: la sectorización de unidades geográficas homogéneas de intervención, identificadas en la base de datos digital confeccionada, y la clasificación de las explotaciones agrícolas en términos de superficie, de acuerdo a los criterios definidos por la Oficina de Estudios y Políticas Agrarias (ODEPA, 2000). En el diseño de la encuesta se consideró un conjunto de variables o parámetros concernientes a: antecedentes generales, aspectos productivos, aspectos financieros y comerciales, y conocimiento sobre el proyecto Convento Viejo II Etapa. El tamaño de la muestra fue de 416 explotaciones, calculado desde un universo de 6.611 unidades, para un nivel de confianza de $95 \%$ y un error de $5 \%$.

La recolección de información en terreno se apoyó con cartografía diseñada especialmente para orientar el recorrido de los encuestadores. Una vez completada la toma de datos, se procedió a elaborar la tipología de las explotaciones, considerando los siguientes aspectos:

\section{- Revisión y selección de variables para el aná-} lisis de tipificación y clasificación: para identificar las variables que efectivamente pudieran contribuir al análisis, se calculó la media, la desviación estándar y el coeficiente de variación (CV) de cada una de ellas, eliminándose aquellas con un CV inferior a 45\%. Luego se calculó una matriz completa de correlaciones entre variables, de manera de identificar aquellas con alta correlación que pudieran destacar un aspecto en particular. Finalmente, se estandarizaron las variables a valores del estadístico T (Everitt y Graham, 2001) para eliminar efectos de magnitud y escalas de las unidades de medida.
- Análisis de componentes principales (ACP): este procedimiento se aplica en estadística multivariada para reducir el número de variables de un problema. El método calcula variables sintéticas denominadas "componentes principales" que se expresan como una combinación lineal de las variables originales. Por medio de este procedimiento se extrajeron 31 componentes principales que dieron cuenta del $75 \%$ de la varianza de la matriz de varianza-covarianza, facilitando la interpretación, visualización y comprensión de las relaciones entre variables.

- Análisis cluster (AC): es una técnica de clasificación jerárquica ascendente en la cual se establecen las distancias entre $\mathrm{p}$ puntos (observaciones) en una nube n-dimensional (n es igual al número de variables) y se procede a la unión, en un mismo cluster o conglomerado, de los puntos más cercanos entre sí. Posteriormente, un cluster se puede unir a otro, llegando a una jerarquía de conglomerados. Para el cálculo de distancias se utilizó la Distancia de Mahalanobis, debido a que este método considera la relación existente entre las variables analizadas (Kaufman y Rousseeuw, 1990). Dicha distancia está definida por:

$$
\mathrm{d}_{\mathrm{ij}}=(1 / \mathrm{Lk})\left(\mathrm{Y}_{\mathrm{i}} \mathrm{k}-\mathrm{Y}_{\mathrm{j}} \mathrm{k}\right)^{2}
$$

donde: $\mathrm{Y}_{\mathrm{i}}=$ vector asociado a la observación $\mathrm{i}$, que contiene los $n$ valores de los n componentes principales; $\mathrm{Y}_{\mathrm{j}}=$ vector asociado a la observación $\mathrm{j}$, que contiene los $\mathrm{n}$ valores de los $\mathrm{n}$ componentes principales; y Lk = varianza asociada al k-ésimo componente principal.

El método de conglomeración utilizado fue el Enlace Promedio, el cual calcula la distancia entre dos puntos, uno en cada grupo, y une en cada paso (nivel jerárquico) los grupos con menor promedio de distancias (Sharma, 1996). El resultado final fue un dendrograma y un calendario de conglomeración con los cuales se pudo identificar los grupos que dieron origen, tras su descripción, a las unidades de producción agrícola.

- Análisis de conglomerados: es una técnica multivariante que permite agrupar variables en función del parecido o similitud existente entre ellas. Presenta la ventaja de detectar el número óptimo de grupos y su composición a partir únicamente de la similitud existente. El método utilizado fue el K-medias, el cual resulta altamente 
útil cuando se trabaja un gran número de variables (Everitt y Graham, 2001). Con este método, se refinaron los resultados obtenidos por medio de la clasificación jerárquica, para obtener la clasificación definitiva de los datos analizados.

\section{Creación del sistema de información geográfica para el área de estudio}

Concluida la codificación, análisis y tipificación de los resultados entregados por la encuesta, se confeccionó una tabla en formato .dbf (database file) conformada por campos (variables consultadas en la encuesta) y registros (valores determinados para cada explotación encuestada) (Rigaux et al., 2002). Posteriormente, se generó una cobertura digital a partir del enlace del rol de propiedad (consultado en terreno y registrado en la tabla) para cada explotación encuestada y el rol del polígono correspondiente, identificado en la cobertura de propiedades del Ministerio de Bienes Nacionales.

A su vez, se procedió a agrupar todas las coberturas digitales tratadas anteriormente, de manera de estructurar la base de datos que alimenta el SIG Convento Viejo, facilitando el acceso y manipula- ción de la información. Para mejorar la visualización y comprensión de cada una de las coberturas, se procedió a categorizar los elementos al interior de cada cobertura, eligiendo una simbología particular para cada caso. Dicha simbología se almacenó como un archivo de extensión .avl (ArcView legend) junto con la cobertura correspondiente en el directorio asignado previamente. Finalmente, se elaboró una interfase SIG que facilita la interacción entre el usuario y la información almacenada en la base de datos, para lo cual se programó cada una de las acciones predeterminadas que debía ejecutar la interfase, empleando Scripts en lenguaje AVENUE (Arctur y Zeiler, 2004). Dichas acciones están dirigidas a otorgar flexibilidad, rapidez y fluidez, en las operaciones de consulta y visualización de la información almacenada y organizada en la base de datos digital.

\section{RESULTADOS Y DISCUSIÓN}

La sectorización de áreas homogéneas permitió apreciar cuatro áreas, que se denominaron: Nuevo Riego, Rinconadas, Mejoramiento del Riego, Seguridad del Riego (Figura 1), las cuales poseen ca-

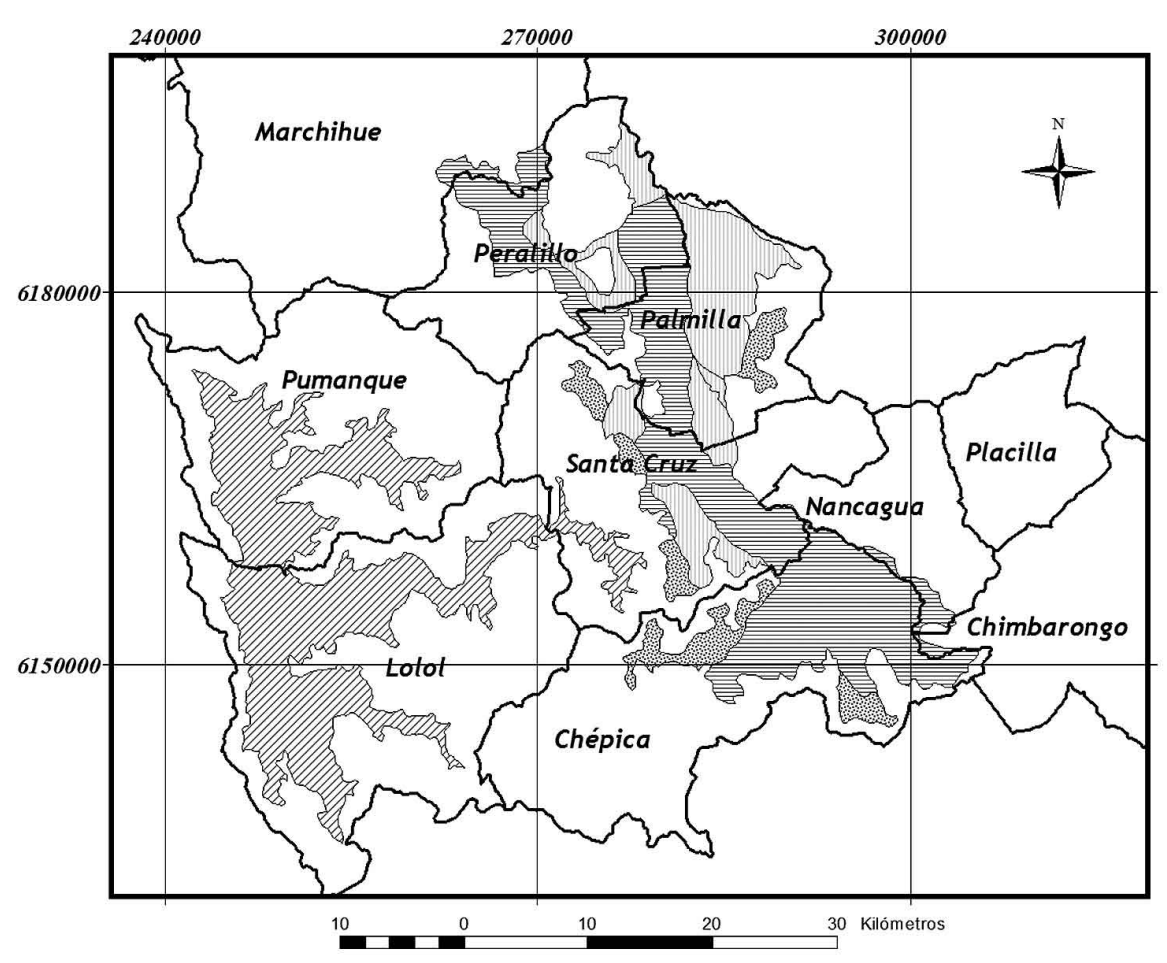

ÁREAS HOMOGÉNEAS Embalse Convento Viejo

\begin{tabular}{l} 
Leyenda \\
Límites comunales \\
Nuevo Riego \\
Minconadas \\
Sistem a de Cooramiento del Riego \\
- Universal Transversal de Mercator \\
- Elipsoide Internacional de 1924 \\
- Datum ProSAD 56 \\
- Zona 19 sur \\
\hline
\end{tabular}

Ubicación Geográfica

Figura 1. Zonas homogéneas dentro del área de estudio.

Figure 1. Homogeneous zones within the study area. 
racterísticas particulares de suelos, clima, uso de suelo y tamaño de explotaciones, que es el resultado que surge a partir del geoprocesamiento digital de las variables geográficas consideradas en el análisis, por lo que su delimitación responde a la suma y combinación de dichas variables expresadas espacialmente.

En el Cuadro 1 se presenta un resumen de las principales variables que caracterizan y diferencian a las áreas homogéneas delimitadas. Como se puede observar, mientras las variables de clima y suelos ofrecen características similares entre los sectores, las variables de tamaño promedio de propiedades y ocupación del suelo son las que determinan una clara diferenciación, lo cual es reflejo de la disponibilidad de agua en cada sector (a mayor disponibilidad de agua, menor superficie por explotación y mayor dedicación a cultivos agrícolas).

En cuanto a la tipología de las explotaciones, obtenida del procesamiento de la encuesta aplicada en terreno, en el Cuadro 2 se presentan los cuatro clusters identificados y los valores observados para cada variable medida; el Cuadro 3 muestra la distribución de los cluster identificados, al interior de la zonas homogéneas. Si se considera el alto promedio de edad de los propietarios (58 años), se podría esperar algunas limitantes en cuanto a la capacidad de recepción de nuevas tecnologías y transformaciones del sistema productivo imperante. La migración familiar es otro factor que no favorece un cambio hacia estructuras de producción más intensivas, en donde se requiere más mano de obra. Sin embargo, un alto porcentaje de encuestados dice tener mano de obra disponible, lo que sumado al alto porcentaje de tenencia de la tierra, podría favorecer una reconversión productiva en un escenario de mayor disponibilidad de agua.

El maíz se presenta como el cultivo de mayor importancia, mientras que el método de riego más usado es el surco, lo cual indica un esquema de producción tradicional que podría variar a un esquema más intensivo orientado a fruticultura y viñedos. La baja capacitación y la falta de información, entre otros, ayudan a explicar el escenario descrito.

Si bien la mayoría de los encuestados expresó tener conocimiento acerca del proyecto de amplia- ción de la capacidad del embalse, no poseen claridad respecto del sistema de concesiones. Esto implica que para asegurar el éxito del proyecto, se deberá realizar una difusión y capacitación técnica, además de dar apoyo financiero, ya que aun cuando se expresó disponibilidad a pagar por el agua, no se cuenta con respaldo económico para enfrentar el nuevo escenario productivo.

La mejora de la infraestructura de riego a realizarse en la zona del Proyecto Convento Viejo II Etapa, deberá ser acompañada de una mejora en la gestión de los recursos que propicie la intensificación, diversificación y especialización de los sistemas de producción. En este sentido, el SIG generado para la zona ofrece un alto nivel de flexibilidad para abordar la situación de cada uno de los sectores de intervención y seguir su desarrollo en el tiempo mediante actualizaciones de fácil ejecución, pudiendo además analizar, simular o proyectar distintas alternativas para el trazado de futuras redes de distribución de agua.

En la Figura 2 se presenta una vista general de la interfase SIG diseñada, la cual consta de tres menús principales destinados a gestionar la información contenida en la base de datos digital del área de estudio. El primero de ellos corresponde a un conjunto de funciones propias del software, con las cuales el usuario puede manipular los archivos en uso. El segundo menú corresponde a submenús desplegables de acceso a las coberturas temáticas almacenadas en la base. El diseño apunta a ofrecer un acceso rápido y organizado a la información, el cual puede ser modificado o ampliado en el futuro, de acuerdo a la disponibilidad de nueva información o a la modificación de la ya existente contenida en la base de datos. Finalmente, el tercer menú principal corresponde a un grupo de botones y herramientas de acceso directo que facilitan el despliegue de las coberturas base (curvas de nivel, hidrografía, caminos, localidades y zonas de estudio). De esta forma, es posible crear una determinada vista incluyendo los temas de interés para el usuario, eliminar dicho arreglo de coberturas, y volver a crear un nuevo arreglo. Con esto la consulta de información se flexibiliza, pudiendo responder a una situación particular de consulta o visualización, en cualquier momento de la sesión de trabajo del operador. 


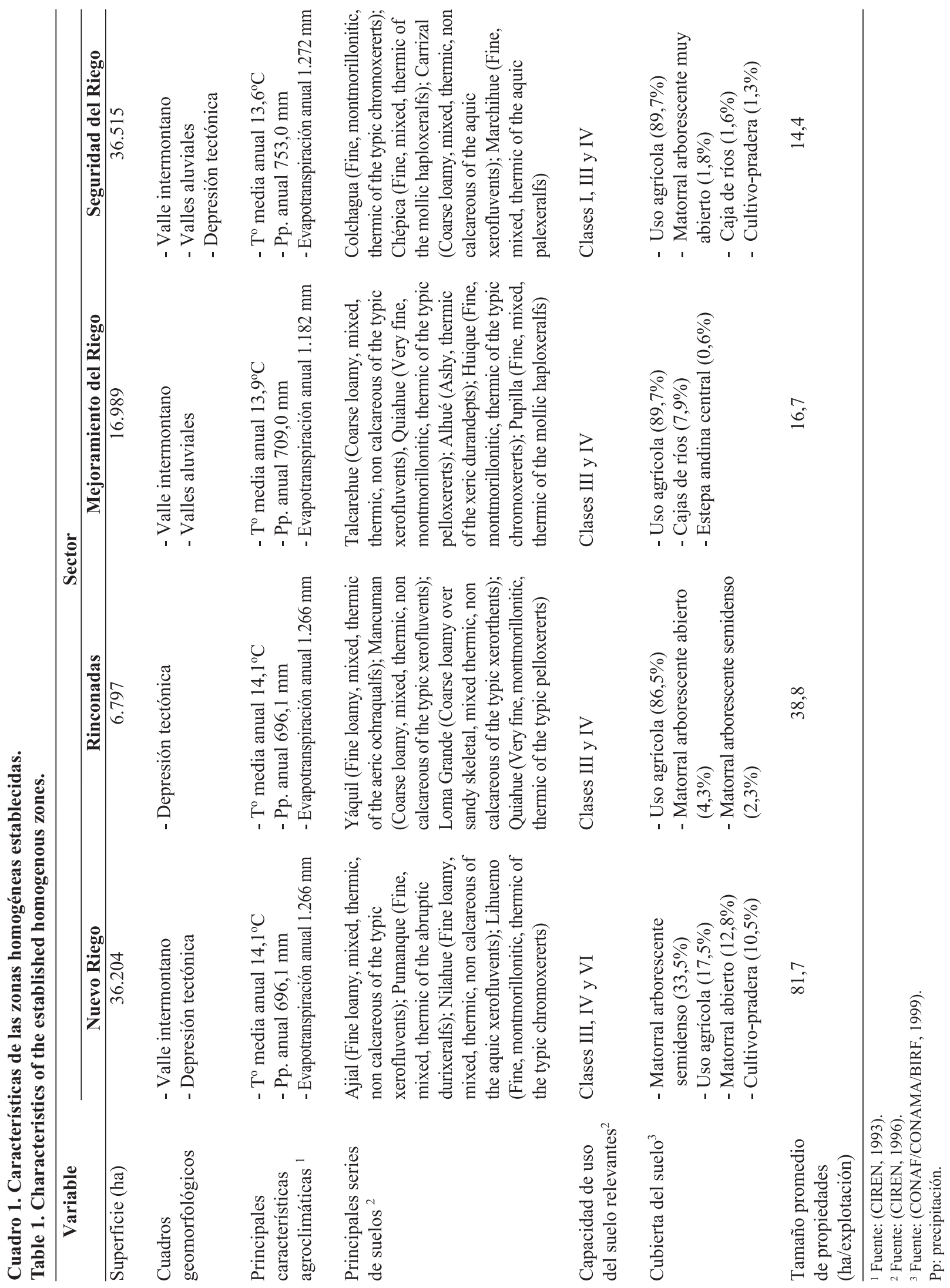




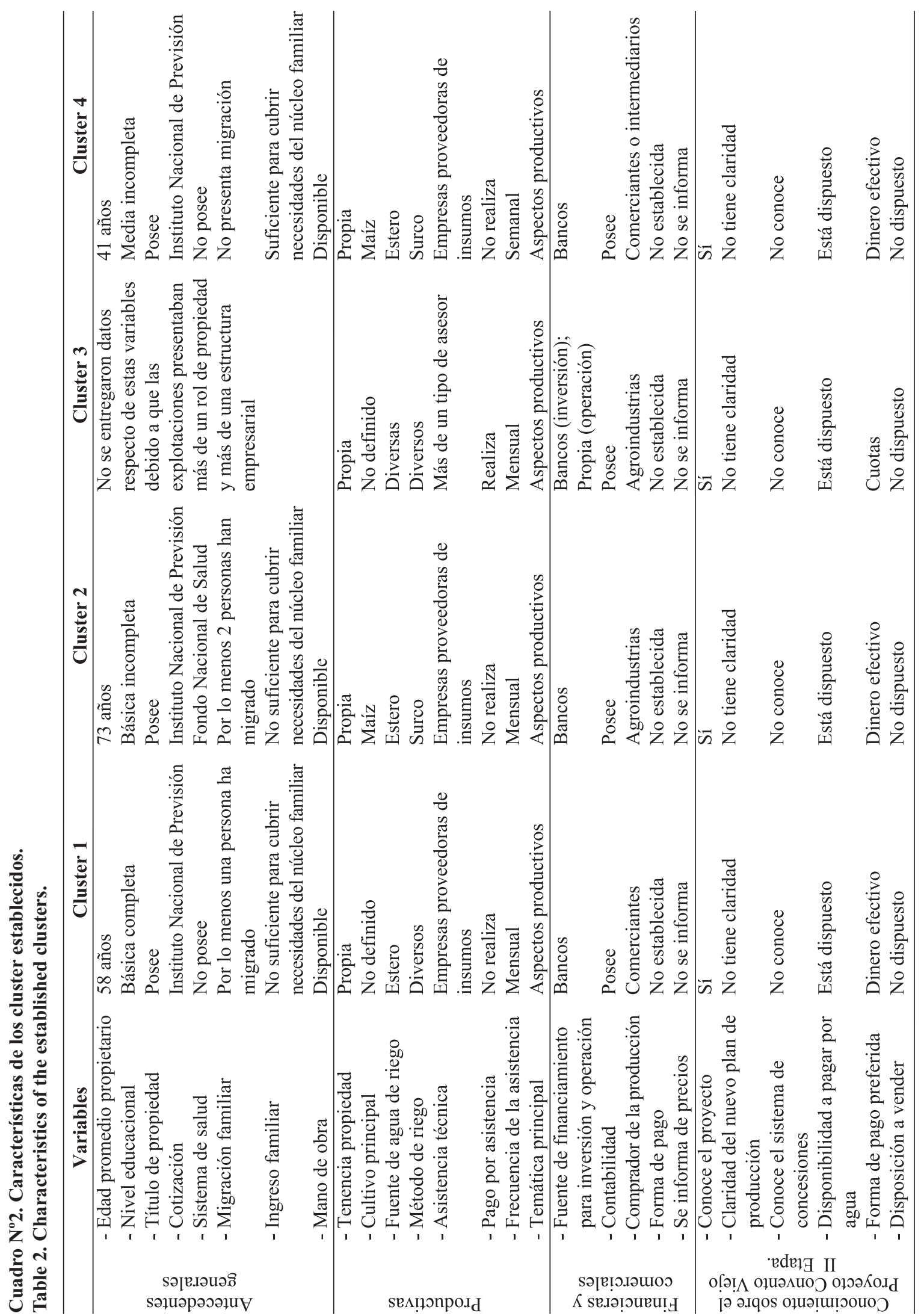


Cuadro 3. Distribución de los cluster dentro de las zonas homogéneas. Table 3. Cluster distribution within the homogeneous zones.

\begin{tabular}{|c|c|c|c|c|c|c|c|c|}
\hline \multirow[t]{2}{*}{ Sector } & \multicolumn{2}{|c|}{ Cluster 1} & \multicolumn{2}{|c|}{ Cluster 2} & \multicolumn{2}{|c|}{ Cluster 3} & \multicolumn{2}{|c|}{ Cluster 4} \\
\hline & $\mathbf{N}^{0}$ & $\%$ & $\mathbf{N}^{0}$ & $\%$ & $\mathbf{N}^{0}$ & $\%$ & $\mathbf{N}^{0}$ & $\%$ \\
\hline Nuevo Riego & 16 & 29,1 & 5 & 9,1 & 18 & 32,7 & 16 & 29,1 \\
\hline Rinconadas & 24 & 52,1 & 14 & 30,4 & 1 & 2,2 & 7 & 15,2 \\
\hline Mejoramiento del Riego & 45 & 56,3 & 23 & 28,8 & 2 & 2,5 & 10 & 12,5 \\
\hline Seguridad del Riego & 88 & 38,9 & 62 & 27,4 & 24 & 10,6 & 52 & 23,0 \\
\hline
\end{tabular}

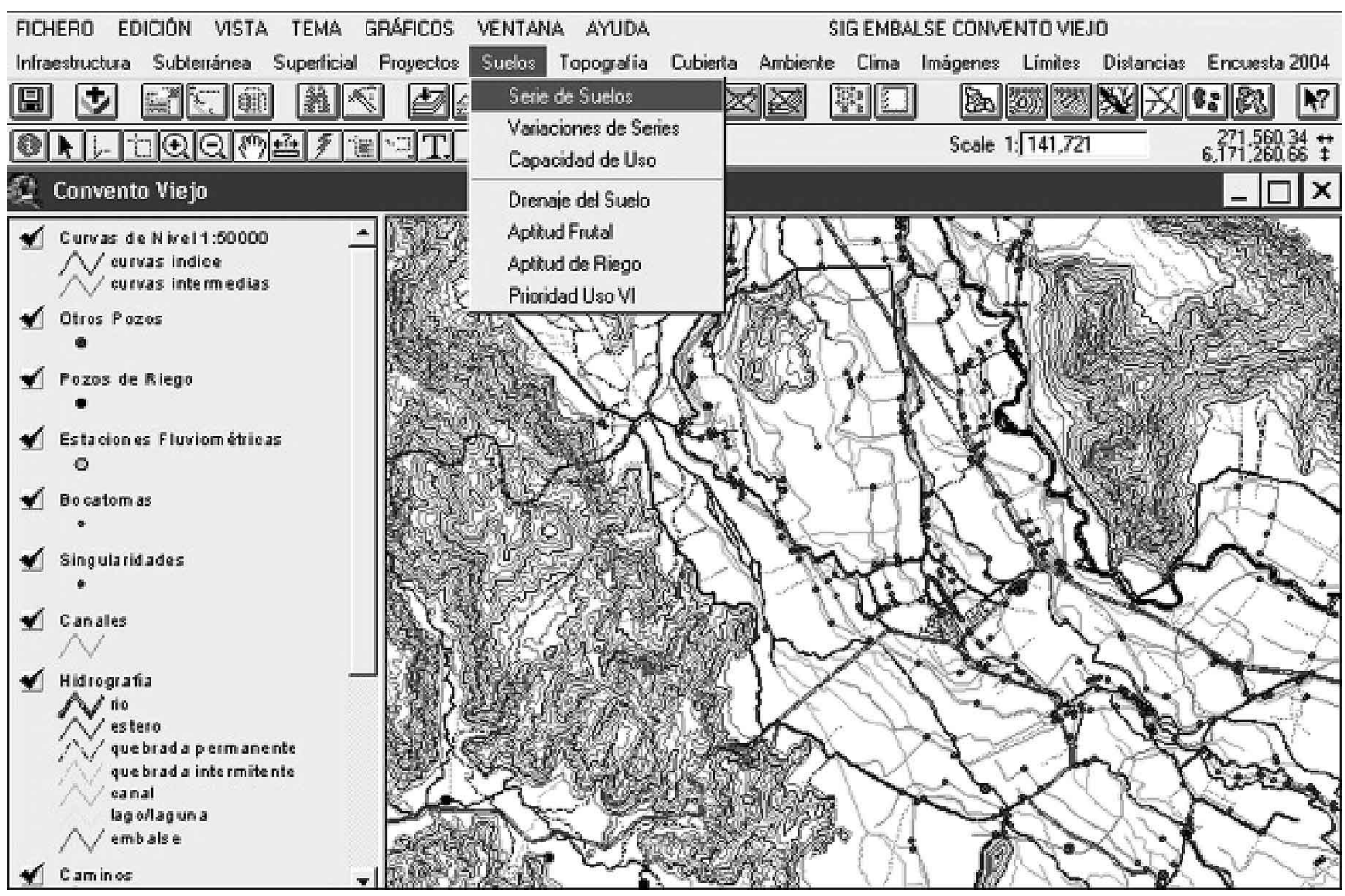

Figura 2. Vista general del Sistema de Información Geográfica (SIG) generado.

Figure 2. Overview of the generated Geographic Information System (GIS).

\section{CONCLUSIONES}

- La zona de estudio se puede dividir en cuatro zonas homogéneas de intervención, de acuerdo a sus características topográficas y agroclimáticas: Nuevo Riego, Rinconadas, Mejoramiento del Riego, Seguridad del Riego.

- Se identificaron cuatro conglomerados (clusters) de explotaciones agrícolas para la zona de estudio, tipificados de acuerdo a variables sociales, productivas, financieras y comerciales.

- La construcción de una base de datos digital georreferenciada agrícola puede incluir informa- ción proveniente de diversas fuentes y formatos, referida a la caracterización de zonas agrícolas de intervención, lo cual permite la estructuración de un panorama preciso y completo de los recursos ambientales y sociales presentes en la zona a intervenir.

- La interfase SIG diseñada permite una fácil consulta y análisis de la información, mejorando la gestión y planificación agrícola del agua de riego al orientar la toma de decisiones en base a la visualización conjunta de variables, la superposición espacial de capas, la determinación de distancias, el despliegue de datos temáticos asociados y la búsqueda selectiva de información. 


\section{LITERATURA CITADA}

Arctur, D., and M. Zeiler. 2004. Designing geodatabases. Case studies in GIS data modeling. 250 p. Esri Press, California, USA.

Bastiaanssen, W., A. Mobin-ud-Din, and T. Zubair. 2003. Upscaling water productivity in irrigated agriculture using remote sensing and GIS technologies. p. 289300. In J.W. Kijne, D. Molden and R. Barker (eds.). Water productivity in agriculture: limits and opportunities for improvement. Comprehensive Assessment of Water Management in Agriculture. Series $N^{\circ}$ 1. CABI Publishing, Wallingford, UK.

Burke, J.J. 2003. Groundwater for irrigation: productivity gains and the need to manage hydro-environmental risk. p. 59-92. In R. Llamas and E. Custodio (eds.). Intensive use of groundwater. Challenges and opportunities. A.A. Balkema Publishers, Lisse, The Netherlands.

CIREN. 1993. Delimitación y descripción de microrregiones para el programa de transferencia tecnológica de INDAP, Regiones V a IX. 99 p. Centro de Información de Recursos Naturales (CIREN), Santiago, Chile.

CIREN. 1996. Estudio agrológico, VI Región. Tomo I y II. Publicación 114. s.p. Centro de Investigación en Recursos Naturales (CIREN), Santiago, Chile.

CONAF/CONAMA/BIRF. 1999. Catastro y evaluación de recursos vegetacionales nativos de Chile. Informe nacional con variables ambientales. 89 p. CONAFCONAMA, Santiago, Chile.

Douglas, D.H. 2000. CONSURF, the Douglas contour to grid methodology. [On line]. Available at http:// www.hig.se/ dds/research/consurf/consur1.htm Accessed 10 January 2004.

Everitt, B., and D. Graham. 2001. Applied multivariate data analysis. 335 p. $2^{\text {nd }}$ ed. Arnold Publishers, New York, USA.

FGDC. 1998. Geospatial positioning accuracy standards. Part 3: National standard for spatial data accuracy. FGDC-STD-007.1-1998. p. 28. Federal Geographic Data Comite (FGDC), Washington D.C., USA.

Hutchinson, S., and L. Daniel. 2000. Inside ArcView GIS. 488 p. $3^{\text {rd }}$ ed. Onword Press, New York, USA.

INE. 1997. Instituto Nacional de Estadísticas [On line]. VI Censo Agropecuario. Disponible en http:// www.ine.cl/34-censo/agropecuario.htm Leído el 7 de enero de 2004.
INE. 2000. Instituto Nacional de Estadísticas [On line]. Disponible en http://www.ine.cl Leído el 7 de enero de 2004.

Kaufman, L., and P.J. Rousseeuw. 2005. Finding groups in data: An introduction to cluster analysis. $368 \mathrm{p} .2^{\text {nd }}$ ed. John Wiley and Sons Inc., New York, USA.

Manz, D. 1994. Evaluación del funcionamiento de los sistemas de conducción de agua para riego, usando modelos de simulación dinámica. 379 p. Uso eficiente del agua. UNESCO/ORCYT (Oficina Regional de Ciencia y Tecnología de la UNESCO para América Latina y el Caribe), Montevideo, Uruguay.

Mena, C. 2005. Geomática para la ordenación del territorio. 316 p. Editorial Universidad de Talca, Talca, Chile.

ODEPA. 2000. Clasificación de las explotaciones agrícolas del VI Censo Nacional Agropecuario según tipo de productor y localización geográfica. Documento de Trabajo $\mathrm{N}^{\circ} 5.45$ p. Oficina de Estudios y Políticas Agrarias (ODEPA), Santiago, Chile.

Rigaux, Ph., D. Scholl, and A. Voisard. 2002. Spatial databases: With application to GIS. 440 p. Morgan Kaufmann Publishers, California, USA.

Sagardoy, J. 2003. Gestión del agua para la agricultura. p. 45-58. III Conferencia. Tecnología para el Desarrollo Humano, Madrid, España. 16 y 17 de octubre. Ingeniería Sin Fronteras, Madrid, España.

Sharma, S. 1996. Applied multivariate techniques. p. 512. John Wiley and Sons Inc., New York, USA.

Van Den Eeckhaut, M., J. Poesen, G. Verstraeten, V. Vanacker, J. Moeyersons, J. Nyssen and L.P.H. Van Beek. 2005. The effectiveness of hillshade maps and expert knowledge in mapping old deep-seated landslides. Geomorphology 67:351-363.

Zapata, A., J. Reca, J.L. Callejón, J.G. López, y J. Martínez. 2000. Predicción del caudal en redes de riego de cultivos intensivos. Revista Riegos y Drenajes XXI. N ${ }^{\circ} 110$. p. 40-45.

Zhang, Q., G. Pavlic, W. Chen, R. Fraser, S. Leblanc and J. Cihlar. 2005. A semi-automatic segmentation procedure for feature extraction in remotely sensed imagery. Computers \& Geosciences. 31:289-296. 